JOURNAL OF INTEGRAL EQUATIONS

AND APPLICATIONS

Volume 4, Number 2, Spring 1992

\title{
EXACT SOLUTION OF A SIMPLE HYPERSINGULAR INTEGRAL EQUATION
}

\author{
P.A. MARTIN
}

ABSTRACT. We obtain the general solution to the simplest one-dimensional hypersingular integral equation; the integral is a Hadamard finite-part integral over a finite interval. We use elementary methods, relating the integral equation to a singular integral equation with a known solution. Despite this, our formula appears to be new.

1. Introduction. We consider the hypersingular integral equation

$$
H f \equiv \frac{1}{\pi} \int_{-1}^{1} \frac{f(t)}{(x-t)^{2}} d t=v(x), \quad-1<x<1 .
$$

Here, $v(x)$ is a known function and $f(x)$ is to be determined. The integral must be interpreted as a Hadamard finite-part integral, defined by

$$
f_{-1}^{1} \frac{f(t)}{(x-t)^{2}} d t=\lim _{\varepsilon \rightarrow 0}\left\{\int_{-1}^{x-\varepsilon} \frac{f(t)}{(x-t)^{2}} d t+\int_{x+\varepsilon}^{1} \frac{f(t)}{(x-t)^{2}} d t-\frac{2 f(x)}{\varepsilon}\right\}
$$

where $|x|<1$ and $f$ is required to have a Hölder-continuous derivative, $f \in C^{1, \alpha}(-1,1)$. The finite-part integral (1.2) is related to a Cauchy principal-value integral by

$$
f_{-1}^{1} \frac{f(t)}{(x-t)^{2}} d t=-\frac{d}{d x} f_{-1}^{1} \frac{f(t)}{x-t} d t
$$

provided that $f \in C^{1, \alpha}$; indeed, (1.3) is sometimes taken as the definition of a finite-part integral. Further properties of finite-part integrals and numerous references to the related literature can be found in $[6,7]$.

In this short paper, we give the general solution of (1.1) for $v$ in a suitably restricted class of functions. This formula seems to be new, and is obtained by exploiting (1.3).

Received by the editors on June 21, 1991 and in revised form on July 3, 1991.

Copyright (C)1992 Rocky Mountain Mathematics Consortium 
The general solution of (1.1) contains two arbitrary constants, which can be determined by imposing two supplementary conditions on the solution, $f$. For example, the integral equation (1.1) arises in potential flow past a thin flat rigid plate. In this context, $f$ represents the discontinuity in the velocity potential for the flow across the plate. Then, the appropriate supplementary conditions are

$$
f(-1)=f(1)=0,
$$

as these ensure that the velocity potential is continuous at the two plate edges $(x= \pm 1)$. With these conditions, we find that

$$
\left(H^{-1} v\right)(x)=\frac{1}{\pi} \int_{-1}^{1} v(t) \log \left(\frac{|x-t|}{1-x t+\sqrt{\left(1-x^{2}\right)\left(1-t^{2}\right)}}\right) d t
$$

The paper concludes with some remarks on (i) closed contours, (ii) pseudo-differential operators, (iii) Chebyshev polynomials, and (iv) non-integrable $v(x)$.

In many applications, (1.1) is generalized to

$$
(H+K) f=v,
$$

where $K$ is another linear operator. Typically,

$$
(K f)(x)=\lambda(x) f(x)+f_{-1}^{1} L(x, t) f(t) d t,
$$

where $\lambda(x)$ is a known function and $L(x, t)$ has an integrable singularity at $x=t$ (perhaps only as a Cauchy principal-value integral). For example, if $L \equiv 0,(1.6)$ is equivalent to Prandtl's equation [8, $\$ 121]$. Numerical methods for the direct treatment of (1.6) are discussed in [7]. Alternatively, one can use $H^{-1}$ to regularize (1.6) as

$$
\left(I+H^{-1} K\right) f=H^{-1} v .
$$

This approach is used in $[\mathbf{2}, \mathbf{3}]$.

2. The airfoil equation. The simplest singular integral equation over a finite interval is the airfoil equation,

$$
\frac{1}{\pi} f_{-1}^{1} \frac{f(t)}{x-t} d t=g(x), \quad-1<x<1 .
$$


This equation and its generalizations have an extensive literature; see, e.g. $[8,9,10,11]$.

If we restrict $g(x)$ to be Hölder continuous for $-1 \leq x \leq 1$, $g \in C^{0, \alpha}[-1,1]$, we can write down the general solution of $(2.1)$; it is (see, e.g. [9, pp. 173-180] or [13, pp. 188-190])

$$
f(x)=\frac{-1}{\pi} f_{-1}^{1} \sqrt{\frac{1-t^{2}}{1-x^{2}}} \frac{g(t)}{x-t} d t+\frac{A}{\sqrt{1-x^{2}}},
$$

where $A$ is an arbitrary constant. Thus, in general, $f(x)$ has an inverse square-root singularity at both $x=-1$ and $x=1$. For a unique solution, we need one supplementary condition on $f$ : often, $f$ is required to be bounded at one end-point.

3. The dominant equation. The simplest hypersingular integral equation over a finite interval is (1.1), which we call the dominant equation. Suppose that $v(x)$ in (1.1) is such that

$$
v(x)=g^{\prime}(x),
$$

where $g \in C^{0, \alpha}[-1,1] \cap C^{1}(-1,1)$; thus $v$ can have integrable end-point singularities. Hence, using (1.3),

$$
\frac{1}{\pi} f_{-1}^{1} \frac{f(t)}{x-t} d t=-g(x)+B
$$

where $B$ is an arbitrary constant of integration. Solving this equation, using (2.2), we obtain

$$
f(x)=\frac{-1}{\pi} f_{-1}^{1} \sqrt{\frac{1-t^{2}}{1-x^{2}}} \frac{B-g(t)}{x-t} d t+\frac{A}{\sqrt{1-x^{2}}} .
$$

Now, apart from another constant of integration, we have

$$
\begin{aligned}
\int \frac{\sqrt{1-t^{2}}}{x-t} d t= & -\sqrt{1-t^{2}}+x \arcsin t \\
& -\sqrt{1-x^{2}} \log \left(\frac{|x-t|}{1-x t+\sqrt{\left(1-x^{2}\right)\left(1-t^{2}\right)}}\right)
\end{aligned}
$$


In particular (cf. (4.3) below, with $n=0$ ),

$$
\frac{1}{\pi} \int_{-1}^{1} \frac{\sqrt{1-t^{2}}}{x-t} d t=x
$$

whence (3.2) gives

$$
f(x)=\frac{1}{\pi} f_{-1}^{1} \sqrt{\frac{1-t^{2}}{1-x^{2}}} \frac{g(t)}{x-t} d t+\frac{A-B x}{\sqrt{1-x^{2}}} .
$$

This is a formula for the general solution of the dominant equation (1.1) in terms of $g$ (which is given by (3.1)). We can obtain a formula in terms of $v$ by an integration by parts, using (3.3); the result is

$$
f(x)=\frac{1}{\pi} \int_{-1}^{1} v(t) \log \left(\frac{|x-t|}{1-x t+\sqrt{\left(1-x^{2}\right)\left(1-t^{2}\right)}}\right) d t+\frac{A+B x}{\sqrt{1-x^{2}}},
$$

where $A$ and $B$ are (new) arbitrary constants.

The first term on the right-hand side of (3.6) is a particular solution of (1.1), for the given function $v$. The second term is the general solution of the homogeneous form of (1.1) (i.e. with $v \equiv 0$ ); it is also given in $[\mathbf{1 0}$, p. 45]. For a unique solution of (1.1), we need two supplementary conditions on $f$; these are often taken to be (1.4) whence $A=B=0$ in (3.6), and (1.5) obtains.

\section{Discussion.}

4.1. Closed contours. We can also consider the dominant equation over a simple, smooth, closed contour $C$, namely

$$
\frac{1}{\pi \mathrm{i}} \oint_{C} \frac{f(t)}{(z-t)^{2}} d t=v(z), \quad z \in C .
$$

The general solution of this integral equation, for continuous $v$, say, is given by

$$
f(z)=\frac{1}{\pi \mathrm{i}} \int_{C} v(t) \log |z-t| d t+A,
$$


where $A$ is an arbitrary constant. For a unique solution of (4.1), we need one supplementary condition on $f$.

4.2. Pseudo-differential operators. It is well known that $H$ is a pseudo-differential operator of order +1 , i.e. $H$ is a continuous linear operator between Sobolev spaces $H^{s} \rightarrow H^{s-1}$; see, e.g. [11, 12]. Thus, roughly speaking, $H$ coarsens by one order. It follows that $H^{-1}$ must be a pseudo-differential operator of order -1 (i.e. smooths by one order), and so we are not surprised to see the kernel $\log |x-t|$ in (1.5), since this gives rise to such an operator [11]; similar remarks apply to (4.1) and (4.2). However, the calculus of pseudo-differential operators does not usually allow one to construct inverse operators explicitly, as done here for the simple operator $H$ supplemented with (1.4).

4.3. Chebyshev polynomials. It is well known that

$$
\frac{1}{\pi} \int_{-1}^{1} \frac{\sqrt{1-t^{2}}}{x-t} U_{n}(t) d t=T_{n+1}(x),
$$

where $T_{n}(x)$ and $U_{n}(x)$ are Chebyshev polynomials of the first and second kinds, respectively. Hence, (1.3) implies that

$$
\frac{1}{\pi} \int_{-1}^{1} \frac{\sqrt{1-t^{2}}}{(x-t)^{2}} U_{n}(t) d t=-(n+1) U_{n}(x) .
$$

Equations (4.3) and (4.4) are valid for $n=0,1,2, \ldots$ and $-1<x<1$. It follows that if

$$
v(x)=U_{n}(x)
$$

in (1.1), then we have

$$
f(x)=\frac{-1}{n+1} \sqrt{1-x^{2}} U_{n}(x),
$$

and (1.4) is satisfied. Since the polynomials $U_{n}$ are orthogonal, satisfying

$$
\int_{-1}^{1} \sqrt{1-t^{2}} U_{m}(t) U_{n}(t) d t=\frac{\pi}{2} \delta_{m n}
$$


we find that

$$
f(x)=\sqrt{1-x^{2}} \sum_{n=0} \frac{v_{n}}{n+1} U_{n}(x)
$$

where

$$
v_{n}=\frac{-2}{\pi} \int_{-1}^{1} \sqrt{1-t^{2}} U_{n}(t) v(t) d t .
$$

This gives an alternative specification of $H^{-1}$, which is used in $[\mathbf{1}, \mathbf{2}$, $\mathbf{3}$. These papers, and [5], also contain applications of the method and additional references.

We note that substitution of (4.5) and (4.6) into (1.5) gives the following identity,

$$
\sqrt{1-x^{2}} U_{n}(x)=-\frac{n+1}{\pi} \int_{-1}^{1} \log \left(\frac{|x-t|}{1-x t+\sqrt{\left(1-x^{2}\right)\left(1-t^{2}\right)}}\right) U_{n}(t) d t,
$$

which is valid for $n=0,1,2, \ldots$ and $|x|<1$. We can rewrite (4.7) as

$$
\int_{-1}^{1} K(x, t) \phi_{n}(t) d t=\lambda_{n} \phi_{n}(x)
$$

where

$$
\phi_{n}(x)=\left(1-x^{2}\right)^{1 / 4} U_{n}(x), \quad \lambda_{n}=\frac{\pi}{n+1}
$$

and

$$
K(x, t)=\frac{1}{\left(1-x^{2}\right)^{1 / 4}\left(1-t^{2}\right)^{1 / 4}} \log \left(\frac{1-x t+\sqrt{\left(1-x^{2}\right)\left(1-t^{2}\right)}}{|x-t|}\right) .
$$

This gives the eigenvalues $\lambda_{n}$ and eigenvectors $\phi_{n}$ of the integral operator with positive, symmetric kernel $K$. Alternatively, if we put $x=\cos \phi$ and $t=\cos \theta$ in (4.7), we obtain

$$
\sin m \phi=\frac{m}{\pi} \int_{0}^{\pi} \log \left|\frac{\sin \frac{\phi+\theta}{2}}{\sin \frac{\phi-\theta}{2}}\right| \sin m \theta d \theta
$$

for $m=1,2, \ldots$, which is equivalent to a known Fourier sine series (use $1.441(2)$ in $[4])$. 
4.4. Non-integrable data. Tricomi $[\mathbf{9}$, p. 181] notes that

$$
\frac{1}{\pi} f_{-1}^{1}\left(\frac{1-t}{1+t}\right)^{\alpha} \frac{d t}{x-t}=\frac{1}{\sin (\pi \alpha)}-\left(\frac{1-x}{1+x}\right)^{\alpha} \cot (\pi \alpha)
$$

for $0<|\alpha|<1$. Differentiating with respect to $x$ gives

$$
\frac{1}{\pi} f_{-1}^{1}\left(\frac{1-t}{1+t}\right)^{\alpha} \frac{d t}{(x-t)^{2}}=-2 \alpha \frac{(1-x)^{\alpha-1}}{(1+x)^{\alpha+1}} \cot (\pi \alpha)
$$

This formula, which is valid for $-1<\alpha<1$, shows that (1.1) has a solution even when $v(x)$ is not integrable at $x=-1(0<\alpha<1)$ or at $x=1(-1<\alpha<0)$. For example, in the first case $(0<\alpha<1)$, there is a solution which is weakly singular at $x=-1$; such solutions cannot be obtained by the methods described in the rest of this paper.

\section{REFERENCES}

1. A. Frenkel, A Chebyshev expansion of singular integrodifferential equations with a $\partial^{2} \ln |s-t| / \partial s \partial t$ kernel, J. Comput. Phys. 51 (1983), 335-342.

2. M.A. Golberg, The convergence of several algorithms for solving integral equations with finite-part integrals, J. Integral Equations 5 (1983), 329-340.

3. - The convergence of several algorithms for solving integral equations with finite part integrals. II, J. Integral Equations 9 (1985), 267-275.

4. I.S. Gradshteyn and I.M. Ryzhik, Table of integrals, series, and products, Academic Press, New York, 1980.

5. A.C. Kaya and F. Erdogan, On the solution of integral equations with strongly singular kernels, Q. Appl. Math. 45 (1987), 105-122.

6. P.A. Martin, End-point behaviour of solutions to hypersingular integral equations, Proc. Roy. Soc. London A 432 (1991), 301-320.

7. - and F.J. Rizzo, On boundary integral equations for crack problems, Proc. Roy. Soc. London A 421 (1989), 341-355.

8. N.I. Muskhelishvili, Singular integral equations, Noordhoff, Groningen, 1953.

9. F.G. Tricomi, Integral equations, Interscience, New York, 1957.

10. E.O. Tuck, Application and solution of Cauchy singular integral equations, in The application and numerical solution of integral equations (R.S. Anderssen, F.R. de Hoog and M.A. Lukas, eds.), Sijthoff and Noordhoff, Alphen aan den Rijn, 1980, 21-49.

11. W.L. Wendland, On applications and the convergence of boundary integral methods, in Treatment of integral equations by numerical methods (C.T.H. Baker and G.F. Miller, eds.), Academic Press, London, 1982, 463-476.

12. - Mathematical properties and asymptotic error estimates for elliptic boundary element methods, in Advanced boundary element methods (T.A. Cruse, ed.), Springer, Berlin 1988, 475-489. 
13. P.P. Zabreyko, et al., Integral equations: A reference text, Noordhoff, Leyden, 1975.

Department of Mathematics, University of Manchester, Manchester M13 9PL, ENGLAND 